\title{
Mercier Stability Improvement in Nonlinear Development of LHD Plasma
}

\author{
Katsuji ICHIGUCHI and Benjamin A. CARRERAS ${ }^{1)}$ \\ National Institute for Fusion Science, 322-6 Oroshi-cho, Toki 509-5292, Japan \\ ${ }^{1)}$ BACV Solutions Inc., 110 Mohawk, Oak Ridge, Tennessee 37831, USA
}

(Received 16 November 2007 / Accepted 10 January 2008)

\begin{abstract}
A stable path to high-beta plasmas is investigated for an inward-shifted LHD configuration. An improvement of Mercier stability is observed due to the nonlinear saturation of the resistive interchange modes. For this study, a multi-scale numerical scheme is used. In this scheme, the beta value is increased by adding a small pressure increment to the background pressure. We focus on the dependence of the Mercier stability on the profile of the pressure increment. It is found that the total pressure profile approaches a profile marginally stable to the Mercier criterion when the pressure increment has a fixed profile.
\end{abstract}

(c) 2008 The Japan Society of Plasma Science and Nuclear Fusion Research

Keywords: MHD, LHD, multi-scale simulation, Mercier criterion, interchange mode, nonlinear saturation, selforganization

DOI: $10.1585 /$ prr.3.S1033

\section{Introduction}

In LHD experiments, good plasma confinement has been observed in the magnetic configuration with the vacuum magnetic axis located at $R_{\mathrm{ax}}=3.6 \mathrm{~m}$ [1]. However, linear ideal interchange modes (Mercier modes) were predicted to be unstable in this configuration [2]. In order to investigate the stabilizing mechanism of the modes, we developed a nonlinear MHD code, NORM, based on the reduced MHD equations [3,4]. In such an investigation, it is crucial to follow the continuous change of the pressure profile with respect to the increase in the beta value. For this purpose, we have also developed a multi-scale simulation scheme [5] by utilizing the NORM code and the VMEC code [6]. This scheme treats both the equilibrium change in the long time scale and the nonlinear dynamics of the instability in the short time scale simultaneously.

In the multi-scale scheme, the beta value is increased by adding a small increment of pressure to the background pressure. In this case, there is freedom in determining the profile of the pressure increment. One choice for the profile is to use the shape similar to the background pressure profile obtained by the nonlinear evolution. In the original study [5], we applied this pressure increment to the inwardshifted LHD configuration. We found a self-organization of the pressure profile, which indicated a stable path to the high-beta regime.

On the other hand, the profiles of heat deposition and particle supply in experiments are usually fixed in the increase of beta. In order to take this situation into account, we consider using a fixed profile for the pressure increment in the present study. We employ two types of increment profiles and compare the results with those of a similar in- crement profile. In particular, we focus on how the Mercier stability is improved by the self-organization of the pressure profile due to the nonlinear saturation of the resistive interchange mode.

This paper is organized as follows. In Section 2, the numerical calculation scheme is discussed. This section is composed of three subsections. In the first subsection, the basic equations and numerical method of the NORM code are reviewed briefly, because the NORM code is the main calculation tool in the numerical scheme. In the second subsection, the choice of the pressure increment in the multi-scale scheme and the evaluation of the Mercier criterion are explained. In the last subsection, the numerical conditions in the application to the LHD plasma are given. In Section 3, the numerical results for the LHD plasma are presented. In particular, the difference in nonlinear evolution of the pressure profile in each pressure increment is discussed. In Section 4, the improvement in the Mercier stability in the saturated state is compared. The relation between the pressure profile formation and the improvement is discussed. Conclusions are given in Section 5.

\section{Numerical Method}

\subsection{Nonlinear calculation with NORM code}

The NORM code is a nonlinear MHD calculation code. The basic equation of the NORM code is the reduced MHD equations for stream function $\Phi$, poloidal magnetic flux $\Psi$, and plasma pressure $P$ [3]. The equations are as follows:

$$
\frac{\partial \Psi}{\partial t}=-\left(\frac{R}{R_{0}}\right)^{2} \mathbf{B} \cdot \nabla \Phi+\frac{1}{S} J_{\zeta},
$$




$$
\begin{aligned}
\frac{\mathrm{d} U}{\mathrm{~d} t}= & \left(\frac{R}{R_{0}}\right)^{2}\left(-\mathbf{B} \cdot \nabla J_{\zeta}+\frac{\beta_{0}}{2 \epsilon^{2}} \nabla_{\perp} \Omega \times \nabla_{\perp} P \cdot \nabla \zeta\right) \\
& +v \hat{\nabla}_{\perp}^{2} U,
\end{aligned}
$$

and

$$
\frac{\mathrm{d} P}{\mathrm{~d} t}=\kappa_{\perp} \Delta_{*} P+\epsilon^{2} \kappa_{\|}\left(\frac{R}{R_{0}}\right)^{2} \mathbf{B} \cdot \nabla(\mathbf{B} \cdot \nabla P) .
$$

Equations (1), (2), and (3) are the Ohm's law, the vorticity equation, and the equation of state, respectively.

The equations are normalized in the following way. The lengths in the $\zeta$ direction and the perpendicular direction are normalized by the major radius $R_{0}$ and the average minor radius $a$ of the plasma, respectively, and $\epsilon$ is given by $a / R_{0}$. Here, $\zeta$ is the toroidal angle. The magnetic field is normalized by the value at the center of the helical coils, $B_{0}$, and $\beta_{0}$ denotes the beta value at the axis. The time is normalized by the Alfvén time $\tau_{\mathrm{A}}$, which is given by

$$
\tau_{\mathrm{A}}=R_{0} \sqrt{\mu_{0} \rho_{\mathrm{m}}} / B_{0},
$$

where $\rho_{\mathrm{m}}$ and $\mu_{0}$ are the mass density and vacuum permeability, respectively.

The magnetic differential operator and the convective time derivative are given by

$$
\mathbf{B} \cdot \nabla=g\left(\frac{R_{0}}{R}\right)^{2} \frac{\partial}{\partial \zeta}-\nabla \Psi \times \nabla \zeta \cdot \nabla
$$

and

$$
\frac{\mathrm{d}}{\mathrm{d} t}=\frac{\partial}{\partial t}+\mathbf{v}_{\perp} \cdot \nabla
$$

respectively. The factor $g$ is introduced to give a correction corresponding to the diamagnetic effect. The perpendicular gradient $\nabla_{\perp}$ is defined as

$$
\nabla_{\perp}=\nabla-\nabla \zeta \frac{\partial}{\partial \zeta}
$$

The perpendicular velocity $\mathbf{v}_{\perp}$, the vorticity $U$, and the toroidal current density $J_{\zeta}$ are defined as

$$
\begin{aligned}
& \mathbf{v}_{\perp}=\left(\frac{R}{R_{0}}\right)^{2} \nabla \Phi \times \nabla \zeta, \\
& U=\hat{\nabla}_{\perp}^{2} \Phi=\left(\frac{R}{R_{0}}\right)^{2} \nabla \cdot \nabla_{\perp} \Phi,
\end{aligned}
$$

and

$$
J_{\zeta}=\Delta_{*} \Psi=\left(\frac{R}{R_{0}}\right)^{2} \nabla \cdot\left(\frac{R_{0}}{R}\right)^{2} \nabla_{\perp} \Psi,
$$

respectively. The magnetic Reynolds number $S$ is defined by $S=\tau_{\mathrm{R}} / \tau_{\mathrm{A}}$, where $\tau_{\mathrm{R}}=\mu_{0} a^{2} / \eta$ is the resistive diffusion time and $\eta$ denotes the resistivity. Viscosity and heat conductivity are also introduced. The viscosity coefficient $v$ is normalized by $a^{2} \rho_{\mathrm{m}} / \tau_{\mathrm{A}}$. The coefficients of perpendicular and parallel heat conductivities, $\kappa_{\perp}$ and $\kappa_{\|}$, are normalized by $a^{2} / \tau_{\mathrm{A}}$.

The term of $\nabla \Omega$ denotes the average field line curvature. This term drives the interchange mode. $\Omega$ is given by

$$
\Omega=\frac{1}{2 \pi} \int_{0}^{2 \pi} \mathrm{d} \zeta\left(\frac{R}{R_{0}}\right)^{2}\left(1+\frac{\left|\mathbf{B}_{\mathrm{eq}}-\overline{\mathbf{B}_{\mathrm{eq}}}\right|^{2}}{B_{0}^{2}}\right),
$$

where the subscript "eq" indicates the equilibrium quantity and the overline denotes the toroidally averaged value.

We utilize the VMEC code [6] for calculating the equilibrium quantities used in the NORM code. The solution of the VMEC code has a three-dimensional geometry, while the reduced equations require an axisymmetric geometry. Thus, we construct the axisymmetric geometry by averaging the VMEC equilibrium solution. In the NORM code, flux coordinates $(\rho, \theta, \zeta)$ are employed in the averaged geometry. Here, $\rho$ denotes the square-root of the normalized toroidal magnetic flux. The poloidal angle $\theta$ is determined so that the equilibrium field line should be explained as a straight line in the $(\theta, \zeta)$ space. As for spatial discretization, the finite difference is employed in the radial direction and the Fourier series are used in the poloidal and toroidal directions. Precise explanation of the formulation and numerical scheme in the NORM code is given in Ref. [3].

\subsection{Multi-scale scheme with fixed pressure increment and evaluation of Mercier cri- terion}

The multi-scale scheme is based on iterative calculations of the nonlinear dynamics and the three-dimensional equilibrium [5]. In this case, we divide the whole calculation time into short intervals. At $t=t^{i}$, the end of the $i$-th interval, we calculate new equilibrium quantities at the higher beta value with the VMEC code. We set them as the equilibrium quantities of $t=t^{i+1}$, the end of the next interval. In order to maintain smooth continuity of the perturbation, we also divide the interval between $t^{i}$ and $t^{i+1}$ into some subintervals and interpolate the equilibrium quantities linearly using the equilibrium quantities of $t=t^{i}$ and $t^{i+1}$. Then, the nonlinear dynamics is calculated for each subinterval with the interpolated equilibrium quantities by the NORM code.

When we calculate the equilibrium with the VMEC code, we incorporate the pressure deformation due to the nonlinear dynamics into the pressure profile. At $t=t^{i}$, the total pressure is obtained as

$$
P_{\text {tot }}^{i}=\langle P\rangle^{i}+\sum_{m \neq 0 \text { or } n \neq 0} \tilde{P}_{m n},
$$

where the tilde indicates a perturbed quantity, and $m$ and $n$ denote the poloidal and the toroidal mode numbers, respectively. Here $\langle P\rangle^{i}$ denotes the average pressure, which is given by

$$
\langle P\rangle^{i}=P_{\mathrm{eq}}^{i}+\tilde{P}_{00}^{i} .
$$


The average pressure includes the effect of the nonlinear dynamics through $\tilde{P}_{00}^{i}$. We calculate $P_{\text {eq }}^{i+1}$ using $\langle P\rangle^{i}$ as

$$
P_{\mathrm{eq}}^{i+1}=\langle P\rangle^{i}+\Delta P^{i+1} \text {. }
$$

Here, $\Delta P^{i+1}$ denotes the pressure increment, which gives the increase in beta. In the original study [5], we employed a similar increment profile given by

$$
\Delta P^{i+1}=\langle P\rangle^{i} \frac{\langle\beta\rangle^{i+1}-\langle\beta\rangle^{i}}{\langle\beta\rangle^{i}},
$$

where $\langle\beta\rangle$ denotes the average beta value. In the present study, we also consider two kinds of fixed profile for the increment given by

$$
\Delta P^{i+1}=P_{\mathrm{IP}}\left(1-\rho^{2}\right)\left(1-\rho^{8}\right)
$$

and

$$
\Delta P^{i+1}=P_{\mathrm{IS}}\left(1-\rho^{2}\right)^{2} .
$$

The factors $P_{\mathrm{IP}}$ and $P_{\mathrm{IS}}$ are adjusted such that the increments of the average beta value of (16) and (17) are same as that of (15), respectively. Hereafter, we call the increments given by (15)-(17) "similar increment," "parabolic increment" and "parabola-squared increment," respectively. This is a first step in incorporating a transport equation in our multi-scale formalism. Here the pressure increment can be interpreted as the result of heating and the different profile increments as different heating source profiles.

In the present analysis, we focus on the change in the Mercier stability in the nonlinear evolution. The Mercier criterion is a local stability criterion for the ideal interchange mode, which is given by [7]

$$
D_{\mathrm{I}}=-\frac{1}{t^{\prime 2}}\left(D_{\mathrm{S}}+D_{\mathrm{W}}+D_{\mathrm{G}}\right)<0
$$

for the stability. Here, each component is given by

$D_{\mathrm{S}}=\frac{1}{4} t^{\prime 2}$

$D_{\mathrm{W}}=P^{\prime}\left(V^{\prime \prime}-P^{\prime}\left\langle\frac{1}{\mathbf{B}^{2}}\right\rangle\right)\left\langle\frac{\mathbf{B}^{2}}{\left|\nabla \Phi_{\mathrm{T}}\right|^{2}}\right\rangle+t^{\prime}\left\langle\frac{\left(I^{\prime} \mathbf{B}-\mathbf{J}\right) \cdot \mathbf{B}}{\left|\nabla \Phi_{\mathrm{T}}\right|^{2}}\right\rangle$

and

$D_{\mathrm{G}}=\left\langle\frac{\mathbf{J} \cdot \mathbf{B}}{\left|\nabla \Phi_{\mathrm{T}}\right|^{2}}\right\rangle^{2}-\left\langle\frac{\mathbf{B}^{2}}{\left|\nabla \Phi_{\mathrm{T}}\right|^{2}}\right\rangle\left\langle\frac{(\mathbf{J} \cdot \mathbf{B})^{2}}{\mathbf{B}^{2}\left|\nabla \Phi_{\mathrm{T}}\right|^{2}}\right\rangle$,

where $t, \Phi_{\mathrm{T}}, I$, and $V$ are the rotational transform, toroidal magnetic flux, net toroidal current, and flux volume, respectively. The prime denotes a derivative with respect to $\Phi_{\mathrm{T}}$ and $\langle f\rangle$ refers to the flux average defined by

$$
\langle f\rangle \equiv \frac{\mathrm{d}}{\mathrm{d} \Phi_{\mathrm{T}}} \int f \mathrm{~d} V .
$$

We evaluate the Mercier criterion at $t=t^{i}$. For the evaluation, we calculate the equilibrium with VMEC code again using the pressure profile of

$$
P_{\mathrm{eq}}^{i}=\langle P\rangle^{i} \text {. }
$$

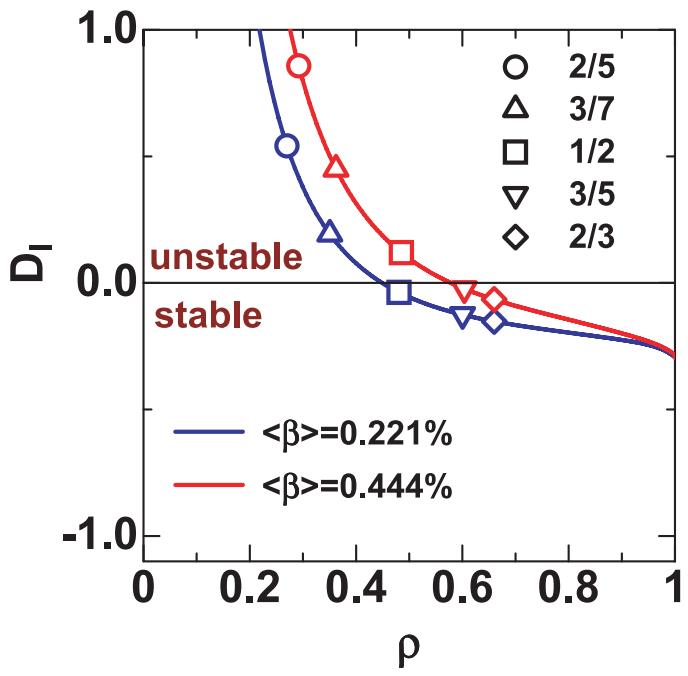

Fig. 1 Profiles of $D_{I}$ of the equilibrium for the pressure profile of $P_{\text {eq }}=P_{0}\left(1-\rho^{2}\right)\left(1-\rho^{8}\right)$ in the inward-shifted LHD plasma. Each symbol is marked at the position of the resonant surface.

\subsection{Application to LHD plasma}

We apply the scheme to the LHD plasma for the three types of pressure increment. We choose the configuration with the vacuum magnetic axis located at $R_{\mathrm{ax}}=3.6 \mathrm{~m}$. We assume the dissipation parameters of $S=10^{6}, v=1.5 \times 10^{-4}, \kappa_{\perp}=1.5 \times 10^{-6}$, and $\epsilon^{2} \kappa_{\|}=$ $1.5 \times 10^{-2}(\epsilon=0.16)$. We examine the evolution for $0.221 \% \leq\langle\beta\rangle \leq 0.498 \%$. One time interval is $2500 \tau_{\mathrm{A}}$, where $\tau_{\mathrm{A}}$ is Alfvén time. We increase the beta value by $\Delta\langle\beta\rangle=0.0138 \%$ after every time interval. In the equilibrium calculation with the VMEC code, we use the freeboundary condition and the no net-current condition. The time interval is divided into ten subintervals for the linear interpolation.

To obtain the initial state, we start from the equilibrium for $P_{\text {eq }}=P_{0}\left(1-\rho^{2}\right)\left(1-\rho^{8}\right)$ at $\langle\beta\rangle=0.221 \%$. As shown in Fig. 1, the core region of $\rho \leq 0.44$ of this equilibrium is Mercier unstable. The absolute value of $D_{\text {I }}$ decreases in the $\rho$ direction. We follow the nonlinear evolution of the resistive interchange mode for this equilibrium and obtain a saturation at $t=10000 \tau_{\mathrm{A}}$. We employ the saturated state as the initial state of the multi-scale calculation and set $t=10000 \tau_{\mathrm{A}}$ as the initial time. Then, the beta value reaches $\langle\beta\rangle=0.498 \%$ at $t=60000 \tau_{\mathrm{A}}$.

\section{Time Evolution of Pressure}

We follow the evolution of the plasma for the three types of increment profile and compare the resultant pressure profiles. First, we discuss the common features in evolution among the increment cases. Figure 2 shows the time evolution of the total kinetic energy for the three pressure increments. It is common that the kinetic energy evolves smoothly compared with the time scale of the subinterval. This feature indicates that the multi-scale approach works 


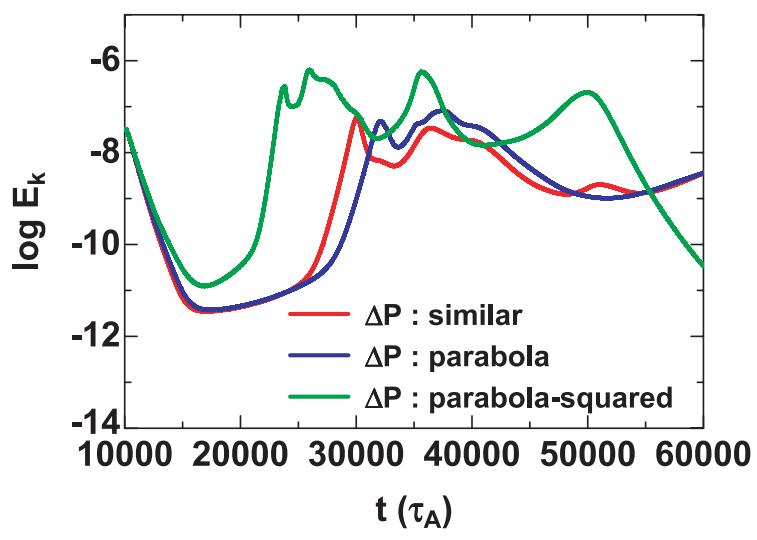

Fig. 2 Time evolution of kinetic energy for each case of the pressure increment.

well in the fixed increment cases as well as in the similar increment case. The evolution of the parabolic increment is close to that of the similar increment, while the evolution of the parabola-squared increment is a little more active.

In the time evolution, excitation and saturation of the resistive interchange mode occur repeatedly in each increment case. Such events can be seen in the time evolution of the total pressure profile and the flow pattern. We show the bird's-eye view of the pressure and the pattern of stream lines at every $10000 \tau_{\mathrm{A}}$ for the parabolic increment case in Fig. 3 as an example. At the initial state, the $(m, n)=$ $(5,2)$ mode is saturated. As time passes, the modes that are unstable at each time are excited but are only mildly saturated. Since the Mercier quantity $D_{\mathrm{I}}$ is a decreasing function of $\rho$, as shown in Fig. 1, the position of the mode excitation moves from the vicinity of the magnetic axis to the outer region in the radial direction. This tendency is the same in other increment cases. In the parabola-squared increment case, the flow of each mode grows more widely; however, there is no significant overlap of the vortices.

Figure 4 shows the bird's-eye view of the total pressure at the final time of $t=60000 \tau_{\mathrm{A}}$ for the cases of the similar and the parabola-squared increments. The deformation of the total pressure is almost independent of $\theta$ for all increment cases including the parabola increment case shown in Fig. 3. This implies that almost all of the resonant resistive interchange modes are saturated at a low level without any significant excitation at this time.

Figure 5 shows the time evolution of the average pressure for each increment cases. The saturation generates local flat structure at the resonant surfaces in the average pressure profile. The flat region is added to the average pressure profile each time a resistive interchange mode is newly saturated.

Next, we discuss the different points that depend on the choice of the increment profile. Remarkable difference in the average pressure profile at the final state is seen in Fig. 5. In the similar increment case, a wide flat structure is generated in the core region of $\rho<0.4$. In the parabolic increment case, the gradient of the pressure is recovered in the region. In the parabola-squared increment case, the gradient becomes larger. These differences are attributed to the gradient of the increment profile.

For any of the increments, the pressure profile is flattened in the core region at $t=30000 \tau_{\mathrm{A}} \sim 40000 \tau_{\mathrm{A}}$, because both $(m, n)=(5,2)$ and $(7,3)$ modes are saturated in this region. In the similar increment case, the average pressure is increased so that the shape should be maintained. Therefore, the local flat structure generated at low beta is almost kept at high beta. On the other hand, in the fixed increment cases, the gradient of the increment profile is always added to the total pressure. Therefore, the local flat structure of the average pressure tends to be smoothed out. Furthermore, the resonant mode can be excited again at the flattened region when the local pressure gradient enhanced by the increment exceeds a critical value. Since the driving force of the mode should be quite weak, the mode saturates immediately to generate a narrower flat region in the average pressure profile. Thus, the local pressure gradient approaches the critical value through this process. The critical value can be measured in terms of $D_{\text {I }}$ as discussed in the next section.

In the parabolic increment case, the increment profile is the same as the equilibrium profile used in the initial state generation. Therefore, the above process is limited in the core region. On the other hand, the steeper gradient is added in the parabola-squared increment case. The region of the process extends to the outer region including the surfaces resonant with the $(5,3)$ and the $(3,2)$ modes.

\section{Mercier Stability Improvement}

The Mercier stability is locally improved by the nonlinear saturation of the resistive interchange mode in all cases of the pressure increments. Figure 6 shows the profiles of $D_{\mathrm{I}}$ at $t=60000 \tau_{\mathrm{A}}(\langle\beta\rangle=0.498 \%)$. In this figure, $D_{\mathrm{I}}$ has negative values around the resonant surfaces corresponding to the local flat pressure regions. On the other hand, the equilibrium with the pressure profile of $P_{\text {eq }}=P_{0}\left(1-\rho^{2}\right)\left(1-\rho^{8}\right)$ at $\langle\beta\rangle=0.444 \%$ is unstable in the whole region of $\rho \leq 0.60$ as shown in Fig. 1. Therefore, the local reduction of the pressure gradient improves the Mercier stability.

The difference in the pressure profile is reflected in the precise structure of $D_{\text {I }}$ profile. In the similar increment case, the improved values of $D_{\mathrm{I}}$ are $-1.05,-0.49$, and -0.21 at resonant surfaces of $t=2 / 5,3 / 7$ and $1 / 2$. There is a tendency that the absolute value is a decreasing function $\rho_{\mathrm{s}}$, where $\rho_{\mathrm{s}}$ is the position of the resonant surfaces. This tendency is related to the Mercier stability at the initial equilibrium. The profile of $D_{\mathrm{I}}$ at $\langle\beta\rangle=0.221 \%$ implies that the driving force of the resistive interchange mode is also the decreasing function of $\rho$. Therefore, the resonant surface at small $\rho_{\mathrm{s}}$ is stabilized earlier than the surface at large $\rho_{\mathrm{s}}$. Since the structure of the pressure pro- 

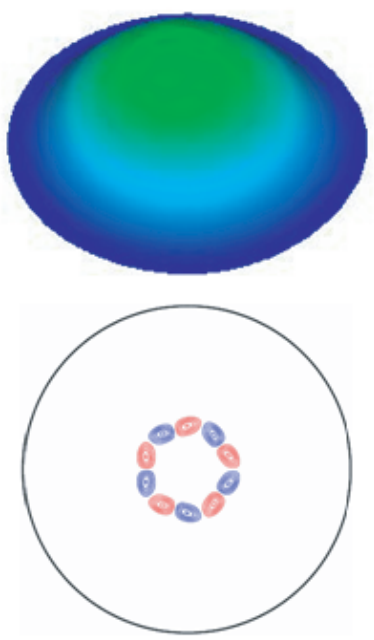

$t=10000 \tau A$
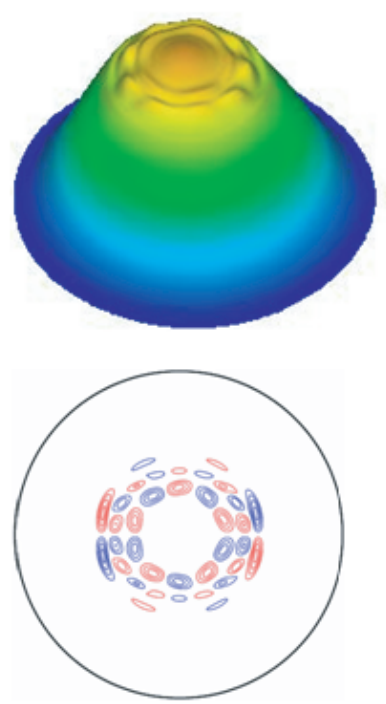

$t=40000 \tau A$
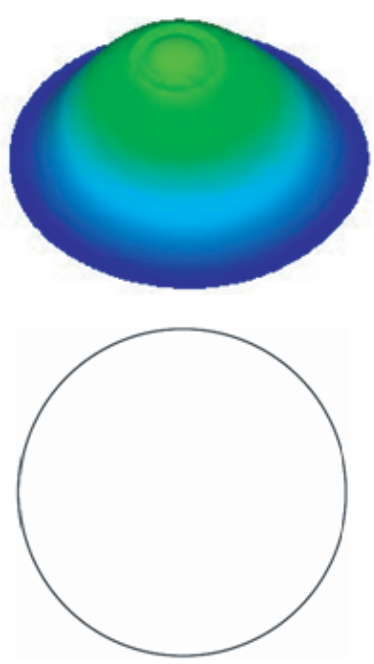

$t=20000 \tau A$
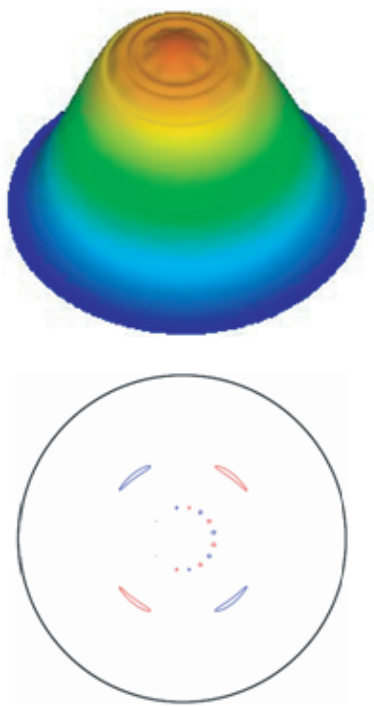

$t=50000 \tau A$
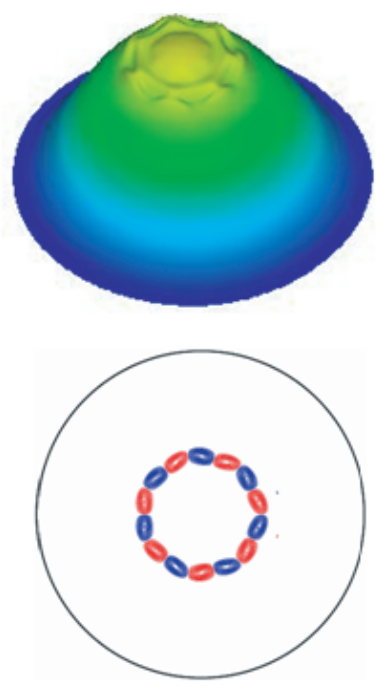

$t=30000 \tau A$
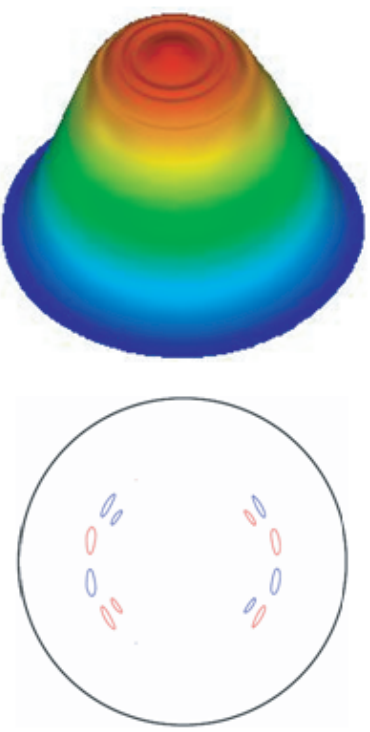

$t=60000 \tau A$

Fig. 3 Time evolution of the total pressure and the flow pattern in the case of parabolic increment.
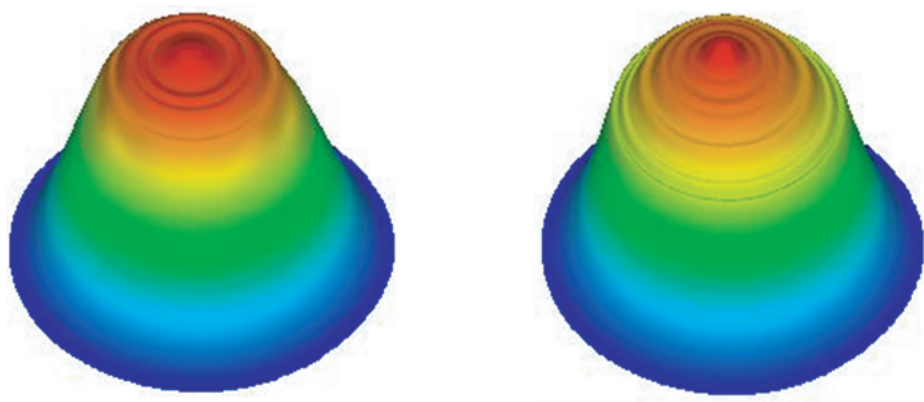

Fig. 4 Bird's-eye view of total pressure at $t=60000 \tau_{\mathrm{A}}$ for similar increment (left) and parabola-squared increment (right). 
(a)

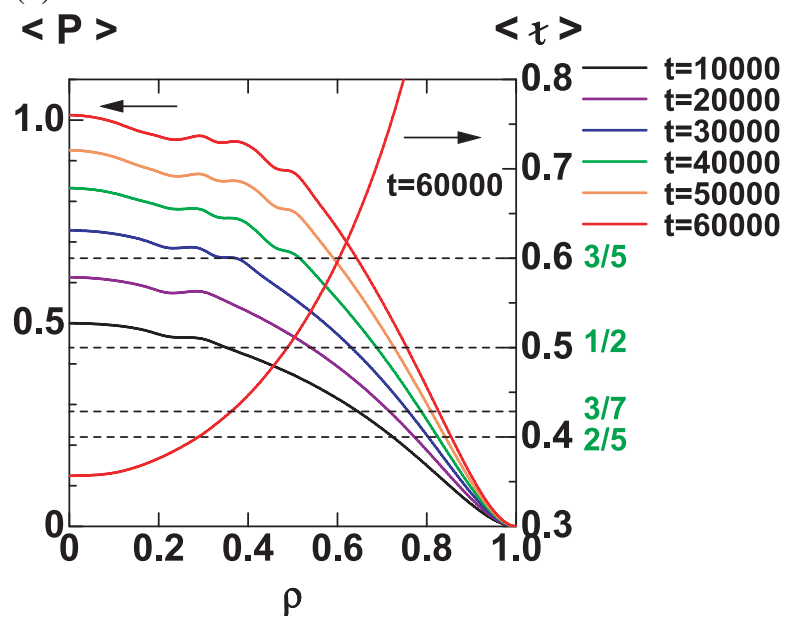

(b)

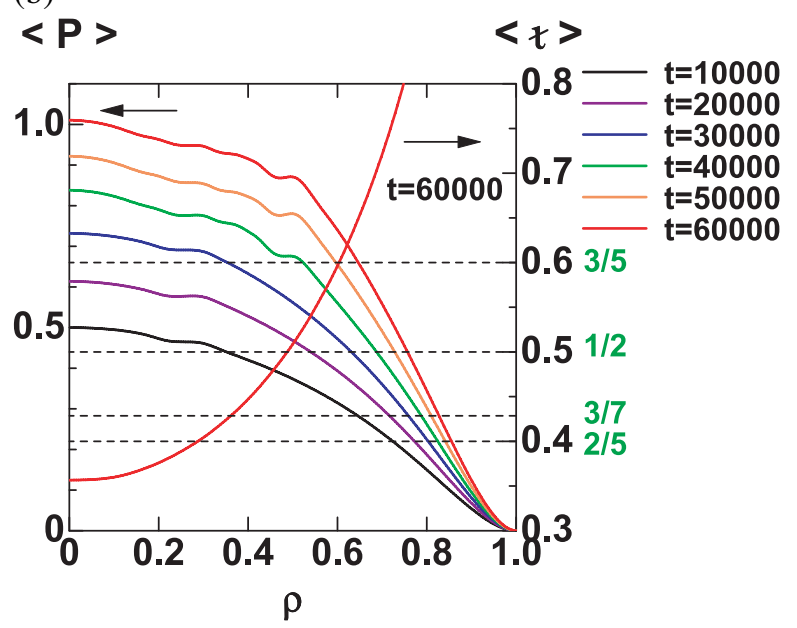

(c)

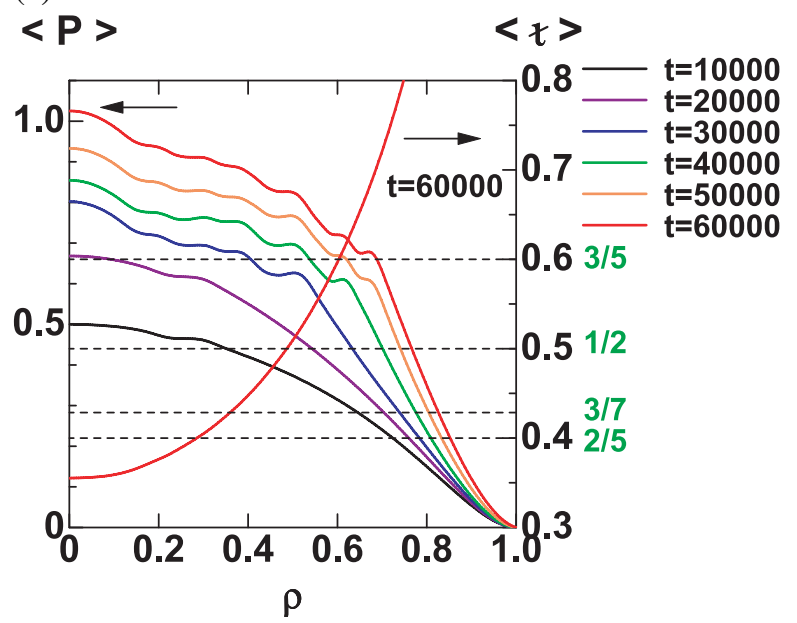

Fig. 5 Time evolution of average pressure in the case of (a) the similar increment, (b) the parabolic increment, and (c) the parabola-squared increment.

file is almost maintained during the beta increase, the resonant surface at small $\rho_{\mathrm{s}}$ becomes more stabilized beyond the marginal stability as the beta value increases.

On the contrary, the absolute value of $D_{\text {I }}$ at all stabilized surfaces is limited in the level of -0.32 in the

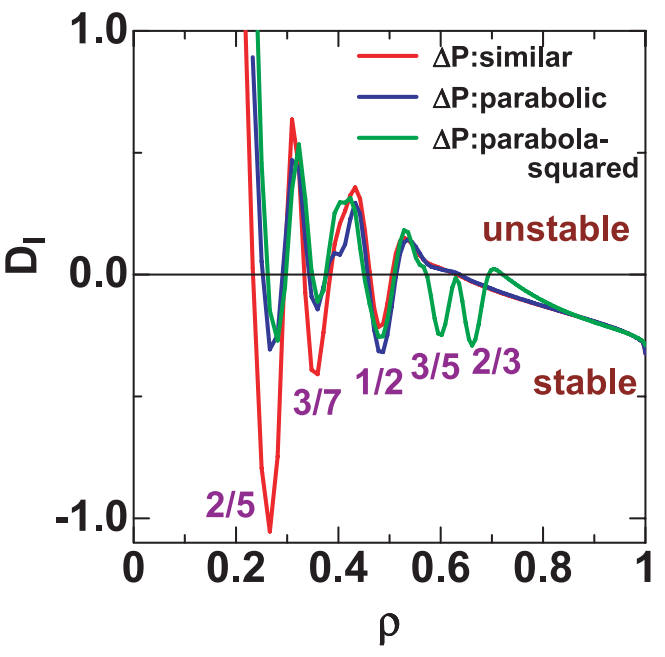

Fig. 6 Profiles of $D_{I}$ at $t=60000 \tau_{\mathrm{A}}$. Numbers show the rotational transform at different positions.

parabolic increment case. In this case, even once the pressure profile is locally flattened, the enhancement of the gradient of the pressure degrades the Mercier stability. Furthermore, the gradient becomes excessively large, and the excitation and saturation of the mode improves the stability again. Therefore, the value of $D_{\text {I }}$ approaches a marginal value in the increase of beta. This tendency is the same as in the case of the parabola-squared increment. In this case, the local improvement is also observed around the surfaces of $t=3 / 5$ and $2 / 3$. Including these surfaces, the absolute value of $D_{\mathrm{I}}$ is limited in the level of -0.29 . The enhancement of the pressure gradient brought by the increment is larger in the parabola-squared increment than in the parabolic increment. Nevertheless, $D_{\mathrm{I}}$ has almost the same value of $D_{\mathrm{I}} \sim-0.3$ at all stabilized surfaces in both the fixed increment cases. This result indicates that this value of $D_{\text {I }}$ corresponds to the marginal pressure gradient independent of the increment profile, if a fixed increment profile is employed. It can be concluded that the local pressure gradient is determined in the increase of beta so that $D_{\text {I }}$ at the resonant surfaces should achieve the marginal value.

\section{Conclusions}

The effect of nonlinear evolution of the resistive interchange mode on the Mercier stability is studied in the inward-shifted LHD configuration. We focus on the dependence of the Mercier criterion on the profile of the pressure increment in the beta increase. The nonlinear saturation generates a local flat region around the resonant surface. Reduction in the pressure gradient at such region results in the local improvement of the Mercier stability. This stabilizing mechanism is common for all of the pressure increment profiles.

However, the radial profile of $D_{\mathrm{I}}$ is different depending on the pressure increment profile. If we use the similar increment profile, the absolute value of negative $D_{\mathrm{I}}$ be- 
comes much larger in the vicinity of the axis than in the outer region. This is attributed to the fact that the locally flat structure in the pressure profile is maintained in the beta increase. On the other hand, if we use a parabolic increment profile, the reduction of the pressure gradient is compensated by the increment pressure. Therefore, the absolute values of $D_{\text {I }}$ at all stabilized surfaces are limited to a small level. In the case of the parabola-squared increment profile, the improvement of the Mercier stability extends to the outer rational surfaces. Even in this case, the absolute values of $D_{\text {I }}$ are also limited to a small level including the outer rational surfaces. The level is almost the same as that in the parabolic increment case. These results indicate that the enhancement and reduction of the pressure gradient are balanced to give a critical pressure gradient. The enhancement is due to addition of the increment pressure and the reduction is due to nonlinear saturation of the mode. That is, in the case of the fixed profile of the pressure increment, the plasma is self-organized so that the pressure profile approaches the marginally stable profile at the resonant surfaces with respect to the Mercier stability.

As a future plan, we consider including an effect of the equilibrium diffusion. In this case, we can expect that the positive $D_{\mathrm{I}}$ values in the regions between the resonant surfaces also approach the marginal value.

\section{Acknowledgments}

This work is supported by NIFS cooperation programs NIFS07KLDD012 and NIFS07KNXN092, and partially supported by the Grant-in-Aid for Scientific Research (C) 17560736 of the Japan Society for the Promotion of Science.

[1] Y. Takeiri et al., Proc. of Joint Conf. 17th Int. Toki Conf. 16th Int. Stellarator/Heliotron Workshop 2007, Oct. 15-19, 2007, Toki, R-01 (2007).

[2] K. Ichiguchi et al., Nucl. Fusion 33, 481 (1993).

[3] K. Ichiguchi et al., Nucl. Fusion 43, 1101 (2003).

[4] K. Ichiguchi et al., Fusion Sci. Technol. 46, 34 (2004).

[5] K. Ichiguchi and B.A. Carreras, J. Plasma Phys. 72, 1117 (2006).

[6] S.P. Hirshman et al., Comput. Phys. Commun. 43, 143 (1986).

[7] A.H. Glasser, J.M. Greene and J.L. Johnson, Phys. Fluids 18, 875 (1975). 